\title{
Mizoguchi-Takahashi's type common fixed point theorems without $T$-weakly commuting condition and invariant approximations
}

\author{
Wutiphol Sintunavarat ${ }^{1 *}$, Dong Min Lee ${ }^{2}$ and Yeol Je Cho 3 , $^{*}$
}

\author{
"Correspondence: \\ wutiphol@mathstat.sci.tu.ac.th \\ yjcho@gnu.ac.kr \\ ${ }^{1}$ Department of Mathematics and \\ Statistics, Faculty of Science and \\ Technology, Thammasat University, \\ Rangsit Center, Pathumthani, 12121, \\ Thailand \\ ${ }^{3}$ Department of Mathematics \\ Education and the RINS, \\ Gyeongsang National University, \\ Jinju, 660-701, Korea \\ Full list of author information is \\ available at the end of the article
}

\begin{abstract}
The purpose of this paper is to introduce a concept of $T_{f}$-orbitally lower semi-continuous mappings which is more general than the concept of $T$-orbitally lower semi-continuous mappings and continuous mappings and also prove Mizoguchi-Takahashi's type coincidence point theorems by using this concept. Moreover, we show that the existence of common fixed points for Mizoguchi-Takahashi's type multi-valued mappings do not require the condition of $T$-weakly commuting mappings. Finally, some invariant approximation results are obtained as applications. Our results unify, extend, and complement several well-known results.

MSC: $47 \mathrm{H} 10 ; 54 \mathrm{H} 25$

Keywords: Mizoguchi-Takahashi's type common fixed point theorem; $T$-weakly commuting mapping; $\mathcal{R}$-function; $\mathcal{M T}$-function; invariant approximation
\end{abstract}

\section{Introduction and preliminaries}

Throughout this paper, we denote by $\mathbb{N}$ the set of all positive integers, by $\mathbb{R}$ the set of all real numbers, and by $\mathbb{R}_{+}$the set of all nonnegative real numbers.

Let $(X, d)$ be a metric space. We denote by $2^{X}$ the class of all nonempty subsets of $X$, by $K(X)$ the class of all nonempty compact subsets of $X$, by $C L(X)$ the class of all nonempty closed subsets of $X$, by $C B(X)$ the class of all nonempty closed bounded subsets of $X$. A functional $H: C L(X) \times C L(X) \rightarrow \mathbb{R}_{+} \cup\{+\infty\}$ is said to be the Pompeiu-Hausdorff generalized metric induced by $d$ is given by

$$
H(A, B)= \begin{cases}\max \left\{\sup _{a \in A} d(a, B), \sup _{b \in B} d(b, A)\right\}, & \text { if the maximum exists; } \\ +\infty, & \text { otherwise }\end{cases}
$$

for all $A, B \in C B(X)$, where $d(a, B)=\inf \{d(a, b): b \in B\}$ is the distance from $a$ to $B \subseteq X$.

Remark 1.1 The following properties of the Pompeiu-Hausdorff generalized metric induced by $d$ are well known:

(1) $H$ is a metric on $C B(X)$

(อ2014 Sintunavarat et al.: licensee Springer. This is an Open Access article distributed under the terms of the Creative Commons Attribution License (http://creativecommons.org/licenses/by/2.0), which permits unrestricted use, distribution, and reproduction in any medium, provided the original work is properly cited. 
(2) If $A, B \in C B(X)$ and $q>1$, then, for all $a \in A$, there exists $b \in B$ such that $d(a, b) \leq q H(A, B)$.

(3) $(C B(X), H)$ is a complete metric space provided $(X, d)$ is a complete metric space.

Definition 1.1 Let $(X, d)$ be a metric space, $f: X \rightarrow X$ and $T: X \rightarrow 2^{X}$ be mappings.

(1) A point $x \in X$ is said to be a fixed point of $f$ (resp., $T$ ) if $x=f x$ (resp., $x \in T x$ ). The set of all fixed points of $f$ (resp., $T$ ) is denoted by $F(f)$ (resp., $F(T)$ ).

(2) A point $x \in X$ is said to be a coincidence point of $f$ and $T$ if $f x \in T x$. The set of all coincidence points of $f$ and $T$ is denoted by $C(f, T)$.

(3) A point $x \in X$ is said to be a common fixed point of $f$ and $T$ if $x=f x \in T x$. The set of all common fixed points of $f$ and $T$ is denoted by $F(f, T)$.

Definition 1.2 Let $(X, d)$ be a metric space, $f: X \rightarrow X$ and $T: X \rightarrow 2^{X}$ be mappings.

(1) If, for any $x_{0} \in X$, there exists a sequence $\left\{x_{n}\right\}$ in $X$ such that $x_{n} \in T x_{n-1}$ for all $n \in \mathbb{N}$, then $O\left(T, x_{0}\right):=\left\{x_{0}, x_{1}, x_{2}, \ldots\right\}$ is said to be an orbit of $T$.

(2) If, for any $x_{0} \in X$, there exists a sequence $\left\{x_{n}\right\}$ in $f(X)$ such that $f x_{n} \in T x_{n-1}$ for all $n \in \mathbb{N}$, then $O_{f}\left(T, x_{0}\right):=\left\{f x_{0}, f x_{1}, f x_{2}, \ldots\right\}$ is said to be an $f$-orbit of $T$.

In 1969, Nadler [1] extended the Banach contraction principle to multi-valued mappings as follows.

Theorem 1.1 (Nadler [1]) Let $(X, d)$ be a complete metric space and $T: X \rightarrow C B(X)$ such that

$$
H(T x, T y) \leq k d(x, y)
$$

for all $x, y \in X$, where $k \in[0,1)$. Then $T$ has at least one fixed point.

Since the theory of multi-valued mappings has many applications in many areas, a number of authors have focused on the topic and have published some interesting fixed point theorems in this frame. Following this trend, in 1972, Reich [2] extended Theorem 1.1 in the following way.

Theorem 1.2 (Reich [2]) Let $(X, d)$ be a complete metric space and $T: X \rightarrow K(X)$ be a mapping satisfying

$$
H(T x, T y) \leq \alpha(d(x, y)) d(x, y)
$$

for all $x, y \in X$, where $\alpha:(0, \infty) \rightarrow[0,1)$ is $R$-function, that is,

$$
\limsup _{x \rightarrow t^{+}} \alpha(x)<1
$$

for all $t \in(0, \infty)$. Then $T$ has at least one fixed point.

Furthermore, Reich [2] also raised the following question in his work:

Can the range of $T$, that $i$, $K(X)$, be replaced by $C B(X)$ or $C L(X)$ ? 
In 1989, Mizoguchi and Takahashi [3] gave the positive answer for the conjecture of Reich [2], when the inequality holds also for $t=0$, as follows.

Theorem 1.3 (Mizoguchi and Takahashi [3]) Let $(X, d)$ be a complete metric space and $T: X \rightarrow C B(X)$. Assume that

$$
H(T x, T y) \leq \alpha(d(x, y)) d(x, y)
$$

for all $x, y \in X$, where $\alpha:[0, \infty) \rightarrow[0,1)$ is MT-function, that is,

$$
\limsup _{x \rightarrow t^{+}} \alpha(x)<1
$$

for all $t \in[0, \infty)$. Then $T$ has at least one fixed point.

Remark 1.2 It is well known that, if $\alpha:[0, \infty) \rightarrow[0,1)$ is a nondecreasing function or a nonincreasing function, then $\alpha$ is a $M T$-function. Therefore, the class of $M T$-functions is a rich class and so this class has been investigated heavily by many authors.

In 2007, Eldred et al. [4] claimed that Theorem 1.3 is equivalent to Theorem 1.1 in the following sense:

If a mapping $T: X \rightarrow C B(X)$ satisfies (1.3), then there exists a nonempty complete subset $M$ of $X$ satisfying the following:

(1) $M$ is $T$-invariant, that is, $T x \subseteq M$ for all $x \in M$.

(2) $T$ satisfies (1.1) for all $x, y \in M$.

In the same year, Suzuki [5] produced an example which shows that MizoguchiTakahashi's fixed point theorem for multi-valued mappings is a real generalization of Nadler's contraction principle. Since the primitive proof of Mizoguchi-Takahashi's fixed point theorem is quite difficult, Suzuki gave a very simple proof of Mizoguchi-Takahashi's theorem. Several authors devoted their attention to investigate its generalizations in various different directions of the Mizoguchi-Takahashi's fixed point theorem (see [6-14] and references therein).

In 2009, Kamran [15] extended the result of Mizoguchi and Takahashi [3] for closed multi-valued mappings and proved a fixed point theorem by using the concept of $T$ orbitally lower semi-continuous mappings as follows:

Definition 1.3 ([16]) Let $(X, d)$ be a metric space, $T: X \rightarrow C L(X)$ be a mapping multivalued, and let $\xi \in X$.

(1) A mapping $g: X \rightarrow \mathbb{R}$ is said to be lower semi-continuous at $\xi$ if, for any sequence $\left\{x_{n}\right\}$ in $X$ such that $x_{n} \rightarrow \xi$ as $n \rightarrow \infty$,

$$
g \xi \leq \liminf _{n \rightarrow \infty} g x_{n}
$$

(2) A mapping $g: X \rightarrow \mathbb{R}$ is said to be $T$-orbitally lower semi-continuous at $\xi$ if, for any sequence $\left\{x_{n}\right\}$ in $O\left(T, x_{0}\right)$ such that $x_{0} \in X$ and $x_{n} \rightarrow \xi$ as $n \rightarrow \infty$,

$$
g \xi \leq \liminf _{n \rightarrow \infty} g x_{n} .
$$


The following result is a main result of Kamran [15].

Theorem 1.4 (Kamran [15]) Let $(X, d)$ be a complete metric space and $T: X \rightarrow C L(X)$ be a mapping satisfying

$$
d(y, T y) \leq \alpha(d(x, y)) d(x, y)
$$

for all $x \in X$ and $y \in T x$, where $\alpha:[0, \infty) \rightarrow[0,1)$ is MT-function. Then:

(K1) For each $x_{0} \in X$, there exist an orbit $\left\{x_{n}\right\}$ of $T$ and $\xi \in X$ such that $\lim _{n \rightarrow \infty} x_{n}=\xi$.

(K2) $\xi$ is a fixed point of $T$ if and only if the function $g: X \rightarrow \mathbb{R}$ defined by $g(x):=d(x, T x)$ for all $x \in X$ is $T$-orbitally lower semi-continuous at $\xi$.

Recently, Ali [17] extended the above result to common fixed point theorem by using the concept of $T$-weakly commuting as follows:

Definition 1.4 ([18]) Let $(X, d)$ be a metric space, $f: X \rightarrow X$ and $T: X \rightarrow C L(X)$ be mappings. The mapping $f$ is said to be $T$-weakly commuting at $x \in X$ if $f f x \in T f x$.

The following result is a main result of Ali [17].

Theorem 1.5 (Ali [17]) Let $(X, d)$ be a metric space, $f: X \rightarrow X$ and $T: X \rightarrow C L(X)$ be two mappings such that $T(X) \subseteq f(X)$ and

$$
d(f y, T y) \leq \alpha(d(f x, f y)) d(f x, f y)
$$

for all $x \in X$ and $f y \in T x$, where $\alpha:[0, \infty) \rightarrow[0,1)$ is MT-function. If $(f(X), d)$ is a complete metric space, then

(A1) For any $x_{0} \in X$, there exists an $f$-orbit $\left\{x_{n}\right\}$ of $T$ and $f \xi \in f(X)$ such that $\lim _{n \rightarrow \infty} f x_{n}=f \xi$.

(A2) $\xi$ is a coincidence point of $f$ and $T$ if and only if the function $h: X \rightarrow \mathbb{R}$ defined by $h(x):=d(f x, T x)$ for all $x \in X$ is lower semi-continuous at $\xi$.

(A3) Ifff $\xi=f \xi$ and $f$ is $T$-weakly commuting at $\xi$, then $f$ and $T$ have a common fixed point.

In this paper, we introduce the concept of $T_{f}$-orbitally lower semi-continuous mappings and, using this concept, prove Mizoguchi-Takahashi's type coincidence point theorems. Also, we show that the condition of ' $T$-weakly commuting of $f$ ' can be omit to prove Mizoguchi-Takahashi's type common fixed point theorems. By the same procedure, we can improve Theorem 1.5 by dropping the condition of ' $f$ is $T$-weakly commuting at $\xi$ ' in (A3). As applications, we derive the invariant approximation results.

\section{Mizoguchi-Takahashi's type coincidence and common fixed point theorems}

In this section, we start with the following concept.

Definition 2.1 Let $(X, d)$ be a metric space, $f: X \rightarrow X, T: X \rightarrow C L(X)$ be mappings, and let $x_{0}, \xi \in X$. 
(1) A mapping $h: f(X) \rightarrow \mathbb{R}$ is said to be lower semi-continuous at $f \xi$ if, for any sequence $\left\{f x_{n}\right\}$ in $f(X)$ such that $f x_{n} \rightarrow f \xi$ as $n \rightarrow \infty$,

$$
h(f \xi) \leq \liminf _{n \rightarrow \infty} h\left(f x_{n}\right)
$$

(2) A mapping $h: f(X) \rightarrow \mathbb{R}$ is said to be $T_{f}$-orbitally lower semi-continuous at $f \xi$ if, for any sequence $\left\{f x_{n}\right\}$ in $O_{f}\left(T, x_{0}\right)$ such that $f x_{n} \rightarrow f \xi$ as $n \rightarrow \infty$,

$$
h(f \xi) \leq \liminf _{n \rightarrow \infty} h\left(f x_{n}\right)
$$

Next, we apply the following useful lemma due to Haghi et al. [19] and Theorem 1.4 to obtain new Mizoguchi-Takahashi’s type common fixed point theorem.

Lemma 2.1 ([19]) Let $X$ be a nonempty set and $f: X \rightarrow X$ be a mapping. Then there exists a subset $E$ of $X$ such that $f(E)=f(X)$ and $\left.f\right|_{E}: E \rightarrow X$ is one-to-one.

The following result is a main result in this paper.

Theorem 2.2 Let $(X, d)$ be a metric space, $f: X \rightarrow X$ and $T: X \rightarrow C L(X)$ be two mappings such that $T(X) \subseteq f(X)$ and

$$
d(f y, T y) \leq \alpha(d(f x, f y)) d(f x, f y)
$$

for all $x \in X$ and $f y \in T x$, where $\alpha:[0, \infty) \rightarrow[0,1)$ is $M T$-function. If $(f(X), d)$ is a complete metric space, then

(S1) For each $x_{0} \in X$, there exist an $f$-orbit $\left\{f x_{n}\right\}$ of $T$ and $f \xi \in f(X)$ such that $\lim _{n \rightarrow \infty} f x_{n}=f \xi$.

(S2) $\xi$ is a coincidence point off and $T$ if and only if the function $h: f(S) \rightarrow \mathbb{R}$ defined by $h(f x):=d(f x, T x)$ for all $f x \in f(S)$ is $T_{f}$-orbitally lower semi-continuous at $f \xi$, where $S \subseteq X$ and $\left.f\right|_{S}$ is one-to-one.

(S3) If $\xi$ is a coincidence point off and $T$ such that ff $\xi=f \xi$, then $f$ and $T$ have a common fixed point.

Proof Let $f: X \rightarrow X$ be a mapping. Using Lemma 2.1, there exists $E \subseteq X$ such that $f(E)=$ $f(X)$ and $\left.f\right|_{E}$ is one-to-one. Now, we can define a mapping $G: f(E) \rightarrow C L(X)$ by

$$
G(f x)=T x
$$

for all $x \in E$. Since $\left.f\right|_{E}$ is one-to-one, it follows that $G$ is well defined. Since $T$ satisfies the contractive condition (2.1), we have

$$
d(f y, T y) \leq \alpha(d(f x, f y)) d(f x, f y)
$$

for all $x \in X$ and $f y \in T x$. By the construction of $G$, we get

$$
d(f y, G(f y)) \leq \alpha(d(f x, f y)) d(f x, f y)
$$


for all $f x \in f(E)$ and $f y \in G(f x)$. This implies that $G$ is satisfies the contractive condition (1.4). From (S1), it follows that, for each $x_{0} \in X$, there exist an orbit $\left\{x_{n}\right\}$ of $G$ and $f \xi \in f(E)$ such that $\lim _{n \rightarrow \infty} f x_{n}=f \xi$. This implies that (K1) in Theorem 1.4 holds.

Again, by the construction of $G$, it follows that (S2) is equivalent to the following condition:

' $f \xi$ is a fixed point of $G$, that is, $f \xi \in G(f \xi)$ if and only if the function $g: f(E) \rightarrow \mathbb{R}$

defined by $g(f x)=d(f x, G(f x))$ for all $f x \in f(E)$ is $G$-orbitally lower semi-continuous at $f \xi$.

Thus (S2) holds. Let $\xi$ is a coincidence point of $f$ and $T$, that is, $f \xi \in T \xi$. Next, we suppose that $f f \xi=f \xi$. Let $z:=f \xi$ and so $z=f \xi=f f \xi=f z \in T \xi$. Since $f z \in T \xi$, it follows from the contractive condition (2.1) that

$$
\begin{aligned}
d(f z, T z) & \leq \alpha(d(f \xi, f z)) d(f \xi, f z) \\
& =\alpha(0) \cdot 0 \\
& =0,
\end{aligned}
$$

which shows that $f z \in T z$. Therefore, $z=f z \in T z$, that is, $z$ is a common fixed point of $f$ and $T$. This completes the proof.

Remark 2.1 Theorem 2.2 generalizes many results in the following sense:

(1) The inequality (2.1) is weaker than some kinds of the contractive conditions such as Mizoguchi-Takahashi's contractive condition [3], Nadler's contractive condition [1], Kamran's contractive condition [15], etc.

(2) The range of $T$ is $C L(X)$ which is more general than $C B(X)$.

(3) For the existence of coincidence point, we merely require that the condition in (S2), whereas other result demands stronger than this condition.

(4) For the existence of common fixed point, we only requires the condition $f f \xi=f \xi$, whereas Theorem 1.5 requires both of this condition and the ' $T$-weakly commuting at $\xi^{\prime}$ condition.

Consequently, Theorem 2.2 extends and improves Nadler's contraction principle [1], Mizoguchi-Takahashi's theorem [3], Theorem 2.1 of Kamran [15], Theorem 2.2 of Ali [17], and several results in the literature. Moreover, for the single-valued case, Theorem 2.2 also unifies Banach's contraction principle [20] and many well-known results.

Corollary 2.3 Let $(X, d)$ be a metric space, $f: X \rightarrow X$ and $T: X \rightarrow C L(X)$ be two mappings such that $T(X) \subseteq f(X)$ and

$$
H(T x, T y) \leq \alpha(d(f x, f y)) d(f x, f y)
$$

for all $x \in X$ and $f y \in T x$, where $\alpha:[0, \infty) \rightarrow[0,1)$ is an MT-function. If $(f(X), d)$ is a complete metric space, then

(S1) For each $x_{0} \in X$, there exist an $f$-orbit $\left\{f x_{n}\right\}$ of $T$ and $f \xi \in f(X)$ such that $\lim _{n \rightarrow \infty} f x_{n}=f \xi$.

(S2) $\xi$ is a coincidence point of $f$ and $T$ if and only if the function $h: f(S) \rightarrow \mathbb{R}$ defined by $h(f x):=d(f x, T x)$ for all $f x \in f(S)$ is $T_{f}$-orbitally lower semi-continuous at $f \xi$, where $S \subseteq X$ and $\left.f\right|_{S}$ is one-to-one. 
(S3) If $\xi$ is a coincidence point of $f$ and $T$ such that ff $\xi=f \xi$, then $f$ and $T$ have a common fixed point.

Proof Since $d(f y, T y) \leq H(T x, T y)$ for all $f y \in T x$, it follows from the contractive condition (2.5) that the inequality (2.1) holds. Therefore, we get the result.

Corollary 2.4 Let $(X, d)$ be a metric space, $f: X \rightarrow X$ and $T: X \rightarrow C L(X)$ be two mappings such that $T(X) \subseteq f(X)$ and

$$
H(T x, T y) \leq \alpha(d(f x, f y)) d(f x, f y)
$$

for all $x, y \in X$, where $\alpha:[0, \infty) \rightarrow[0,1)$ is an MT-function. If $(f(X), d)$ is a complete metric space, then:

(S1) For each $x_{0} \in X$, there exist an $f$-orbit $\left\{f x_{n}\right\}$ of $T$ and $f \xi \in f(X)$ such that $\lim _{n \rightarrow \infty} f x_{n}=f \xi$.

(S2) $\xi$ is a coincidence point of $f$ and $T$ if and only if the function $h: f(S) \rightarrow \mathbb{R}$ defined by $h(f x):=d(f x, T x)$ for all $f x \in f(S)$ is $T_{f}$-orbitally lower semi-continuous at $f \xi$, where $S \subseteq X$ and $\left.f\right|_{S}$ is one-to-one.

(S3) If $\xi$ is a coincidence point of $f$ and $T$ such that ff $\xi=f \xi$, then $f$ and $T$ have a common fixed point.

Proof Since the condition (2.6) implies the condition (2.5), we get the result.

If we take $\alpha(t)=k$ for all $t \in[0, \infty)$, where $k$ is constant number with $k \in[0,1)$, then we get the following result.

Corollary 2.5 Let $(X, d)$ be a metric space, $f: X \rightarrow X$ and $T: X \rightarrow C L(X)$ be two mappings such that $T(X) \subseteq f(X)$ and satisfying

$$
d(f y, T y) \leq k d(f x, f y)
$$

for each $x \in X$ and $f y \in T x$, where $k \in[0,1)$. If $(f(X), d)$ is a complete metric space, then:

(S1) For each $x_{0} \in X$, there exist an $f$-orbit $\left\{x_{n}\right\}$ of $T$ and $f \xi \in f(X)$ such that $\lim _{n \rightarrow \infty} f x_{n}=f \xi$.

(S2) $\xi$ is a coincidence point of $f$ and $T$ if and only if the function $h: f(S) \rightarrow \mathbb{R}$ defined by $h(f x):=d(f x, T x)$ for all $f x \in f(S)$ is $T_{f}$-orbitally lower semi-continuous at $f \xi$, where $S \subseteq X$ and $\left.f\right|_{S}$ is one-to-one.

(S3) If $\xi$ is a coincidence point of $f$ and $T$ such that ff $\xi=f \xi$, then $f$ and $T$ have $a$ common fixed point.

Corollary 2.6 Let $(X, d)$ be a metric space, $f: X \rightarrow X$ and $T: X \rightarrow C L(X)$ be two mappings such that $T(X) \subseteq f(X)$ and

$$
H(T x, T y) \leq k d(f x, f y)
$$

for all $x \in X$ and $f y \in T x$, where $k \in[0,1)$. If $(f(X), d)$ is a complete metric space, then: 
(S1) For each $x_{0} \in X$, there exist an $f$-orbit $\left\{f x_{n}\right\}$ of $T$ and $f \xi \in f(X)$ such that $\lim _{n \rightarrow \infty} f x_{n}=f \xi ;$

(S2) $\xi$ is a coincidence point of $f$ and $T$ if and only if the function $h: f(S) \rightarrow \mathbb{R}$ defined by $h(f x):=d(f x, T x)$ for all $f x \in f(S)$ is $T_{f}$-orbitally lower semi-continuous at $f \xi$, where $S \subseteq X$ and $\left.f\right|_{S}$ is one-to-one;

(S3) If $\xi$ is a coincidence point off and $T$ such that ff $\xi=f \xi$, then $f$ and $T$ have a common fixed point.

Corollary 2.7 Let $(X, d)$ be a metric space, $f: X \rightarrow X$ and $T: X \rightarrow C L(X)$ be two mappings such that $T(X) \subseteq f(X)$ and

$$
H(T x, T y) \leq k d(f x, f y)
$$

for all $x, y \in X$, where $k \in[0,1)$. If $(f(X), d)$ is a complete metric space, then:

(S1) For each $x_{0} \in X$, there exist an $f$-orbit $\left\{f x_{n}\right\}$ of $T$ and $f \xi \in f(X)$ such that $\lim _{n \rightarrow \infty} f x_{n}=f \xi$

(S2) $\xi$ is a coincidence point off and $T$ if and only if the function $h: f(S) \rightarrow \mathbb{R}$ defined by $h(f x):=d(f x, T x)$ for all $f x \in f(S)$ is $T_{f}$-orbitally lower semi-continuous at $f \xi$, where $S \subseteq X$ and $\left.f\right|_{S}$ is one-to-one.

(S3) If $\xi$ is a coincidence point of $f$ and $T$ such that ff $\xi=f \xi$, then $f$ and $T$ have $a$ common fixed point.

\section{Invariant approximation results}

Several problems concerning invariant approximations for self-mappings were obtained as applications of fixed point, coincidence point, and common fixed point results (see [21-27] and references therein). Also, Kamran [18], Latif and Bano [28], and O'Regan and Shahzad $[29,30]$ obtained invariant approximation results for multi-valued mappings.

In this section, we study invariant approximation results for nonlinear single-valued mapping and multi-valued mapping as applications of main results in Section 2.

Let $M$ be a subset of a normed space $E$ and $p \in E$. The set

$$
\operatorname{Best}_{M}(p):=\{x \in M:\|x-p\|=d(p, M)\}
$$

is called the set of best $M$-approximants to $p \in X$ out of $M$, where $d(p, M)=\inf _{y \in M}\|y-p\|$.

Here, we derive some invariant approximation results.

Theorem 3.1 Let $M$ be subset of normed space $(E,\|\cdot\|), p \in E, f: M \rightarrow M$ be a mapping and $T: M \rightarrow C L(M)$ be a multi-valued mappings such that

$$
d(f y, T y) \leq \alpha(\|f x-f y\|)\|f x-f y\|
$$

for each $x \in B_{M}(p)$ and $f y \in T x$, where $\alpha:[0, \infty) \rightarrow[0,1)$ is an MT-function. Suppose that the following conditions hold:

(1) $T\left(\operatorname{Best}_{M}(p)\right) \subseteq f\left(\operatorname{Best}_{M}(p)\right)=\operatorname{Best}_{M}(p)$.

(2) $f\left(\operatorname{Best}_{M}(p)\right)$ is a complete subspace of $M$.

(3) $\left.f\right|_{\text {Best }_{M}(p)}$ is one-to-one.

(4) $\sup _{y \in T x}\|y-p\| \leq\|f x-p\|$ for all $x \in \operatorname{Best}_{M}(p)$. 
Then we have the following:

(S1) For each $x_{0} \in \operatorname{Best}_{M}(p)$, there exists an $f$-orbit $\left\{f x_{n}\right\}$ of $T$ and $f \xi \in f\left(\operatorname{Best}_{M}(p)\right)$ such that $\lim _{n \rightarrow \infty} f x_{n}=f \xi$.

(S2) $\xi \in C(f, T) \cap \operatorname{Best}_{M}(p)$ if and only if the function $h: f\left(\operatorname{Best}_{M}(p)\right) \rightarrow \mathbb{R}$ defined by $h(f x):=d(f x, T x)$ for all $f x \in f\left(\operatorname{Best}_{M}(p)\right)$ is $T_{f}$-orbitally lower semi-continuous at $f \xi$.

(S3) If $\xi \in C(f, T) \cap \operatorname{Best}_{M}(p)$ such that ff $\xi=f \xi$, then $f \xi \in F(f, T) \cap \operatorname{Best}_{M}(p)$.

Proof From the assumption, it follows that $\left.f\right|_{\operatorname{Best}_{M}(p)}$ is a single-valued mapping from $\operatorname{Best}_{M}(p)$ to $\operatorname{Best}_{M}(p)$. Now, we show that $\left.T\right|_{\text {Best }_{M}(p)}$ is a multi-valued mapping from $\operatorname{Best}_{M}(p)$ to $C L\left(\operatorname{Best}_{M}(p)\right)$. First, we claim that $T x \subseteq \operatorname{Best}_{M}(p)$ for all $x \in \operatorname{Best}_{M}(p)$. Let $x \in \operatorname{Best}_{M}(p)$ and $z \in T x$. Since $f\left(\operatorname{Best}_{M}(p)\right)=\operatorname{Best}_{M}(p)$, we have $f x \in \operatorname{Best}_{M}(p)$ and hence $\|f x-p\|=d(p, M)$.

Now, we obtain

$$
d(p, M) \leq\|z-p\| \leq \sup _{y \in T x}\|y-p\| \leq\|f x-p\|=d(p, M) .
$$

This implies that $\|z-p\|=d(p, M)$ and thus $z \in \operatorname{Best}_{M}(p)$. Therefore, $T x \subseteq \operatorname{Best}_{M}(p)$ for all $x \in \operatorname{Best}_{M}(p)$. Since $T x$ is closed for all $x \in M$, it follows that $T x$ is closed for all $x \in \operatorname{Best}_{M}(p)$. Hence $\left.T\right|_{\operatorname{Best}_{M}(p)}$ is a multi-valued mapping from $\operatorname{Best}_{M}(p)$ to $C L\left(\operatorname{Best}_{M}(p)\right)$. It is easy to obtain that

$$
F\left(\left.f\right|_{\operatorname{Best}_{M}(p)},\left.T\right|_{\operatorname{Best}_{M}(p)}\right)=F(f, T) \cap \operatorname{Best}_{M}(p) .
$$

Thus the result follows from Theorem 2.2 with $X=\operatorname{Best}_{M}(p)$. This completes the proof.

Theorem 3.2 Let $M$ be subset of normed space $(E,\|\cdot\|), p \in E$, and $T: M \rightarrow C L(M)$ be a multi-valued mapping such that

$$
d(y, T y) \leq \alpha(\|x-y\|)\|x-y\|
$$

for all $x \in \operatorname{Best}_{M}(p)$ and $y \in T x$, where $\alpha:[0, \infty) \rightarrow[0,1)$ is an MT-function. Suppose that the following conditions hold:

(1) $T\left(\operatorname{Best}_{M}(p)\right) \subseteq \operatorname{Best}_{M}(p)$;

(2) $\mathrm{Best}_{M}(p)$ is complete subspace of $M$;

(3) $\sup _{y \in T x}\|y-p\| \leq\|x-p\|$ for all $x \in \operatorname{Best}_{M}(p)$.

Then we have the following:

(S1) For each $x_{0} \in \operatorname{Best}_{M}(p)$, there exists an orbit $\left\{x_{n}\right\}$ of $T$ and $\xi \in \operatorname{Best}_{M}(p)$ such that $\lim _{n \rightarrow \infty} x_{n}=\xi$

(S2) $\xi \in F(T) \cap \operatorname{Best}_{M}(p)$ if and only if the function $g:$ Best $_{M}(p) \rightarrow \mathbb{R}$, defined by $g(x):=d(x, T x)$ for all $x \in f\left(\operatorname{Best}_{M}(p)\right)$, is $T$-orbitally lower semi-continuous at $\xi$.

Proof Take $f$ as the identity mapping from $M$ into $M$ in Theorem 3.1, we get the result. 


\section{Competing interests}

The authors declare that they have no competing interests.

\section{Authors' contributions}

All authors contributed equally and significantly in writing this paper. All authors read and approved the final manuscript.

\section{Author details}

'Department of Mathematics and Statistics, Faculty of Science and Technology, Thammasat University, Rangsit Center Pathumthani, 12121, Thailand. ²Department of Mathematics, Gyeongsang National University, Jinju, 660-701, Korea.

${ }^{3}$ Department of Mathematics Education and the RINS, Gyeongsang National University, Jinju, 660-701, Korea.

${ }^{4}$ Department of Mathematics, Faculty of Science, King Abdulaziz University, Jeddah, Saudi Arabia.

\section{Acknowledgements}

The third author was supported by the Basic Science Research Program through the National Research Foundation of Korea funded by the Ministry of Education, Science and Technology (KRF-2013053358).

\section{Received: 9 February 2014 Accepted: 23 April 2014 Published: 08 May 2014}

\section{References}

1. Nadler, SB Jr: Multi-valued contraction mappings. Pac. J. Math. 30, 475-488 (1969)

2. Reich, S: Fixed points of contractive functions. Boll. Unione Mat. Ital. 4(5), 26-32 (1972)

3. Mizoguchi, N, Takahashi, W: Fixed point theorems for multivalued mappings on complete metric spaces. J. Math. Anal. Appl. 141, 177-188 (1989)

4. Eldred, AA, Anuradha, J, Veeramani, P: On equivalence of generalized multi-valued contractions and Nadler's fixed point theorem. J. Math. Anal. Appl. 336, 751-757 (2007)

5. Suzuki, T: Mizoguchi-Takahashi's fixed point theorem is a real generalization of Nadler's. J. Math. Anal. Appl. 340 752-755 (2008)

6. Ali, MU, Kamran, T: Hybrid generalized contractions. Math. Sci. 7, Article ID 29 (2013)

7. Du, W-S: Coupled fixed point theorems for nonlinear contractions satisfied Mizoguchi-Takahashi's condition in quasiordered metric spaces. Fixed Point Theory Appl. 2010, Article ID 876372 (2010)

8. Du, W-S: On coincidence point and fixed point theorems for nonlinear multivalued maps. Topol. Appl. 159, 49-56 (2012)

9. Kamran, T: Fixed point theorems for hybrid mappings satisfying an integral type contractive condition. Georgian Math. J. 19, 117-125 (2012)

10. Lin, I-J, Chen, T-H: New existence theorems of coincidence points approach to generalizations of Mizoguchi-Takahashi's fixed point theorem. Fixed Point Theory Appl. 2012, Article ID 156 (2012)

11. Shatanawi, W, Ćojbašic Rajić, V, Radenović, S, Al-Rawashdeh, A: Mizoguch-Takahashi-type theorems in tvs-cone metric spaces. Fixed Point Theory Appl. 2012, Article ID 106 (2012)

12. Sintunavarat, W, Kumam, P: Weak condition for generalized multi-valued $(f, \alpha, \beta)$-weak contraction mappings. Appl. Math. Lett. 24, 460-465 (2011)

13. Sintunavarat, W, Kumam, P: Common fixed point theorems for hybrid generalized multi-valued contraction mappings. Appl. Math. Lett. 25, 52-57 (2012)

14. Sintunavarat, W, Kumam, P: Common fixed point theorem for cyclic generalized multi-valued contraction mappings. Appl. Math. Lett. 25, 1849-1855 (2012)

15. Kamran, T: Mizoguchi-Takahashi's type fixed point theorem. Comput. Math. Appl. 57, 507-511 (2009)

16. Hicks, TL, Rhoades, BE: A Banach type fixed point theorem. Math. Jpn. 24, 327-330 (1979)

17. Ali, MU: Mizoguchi-Takahashi's type common fixed point theorem. J. Egypt. Math. Soc. (2013, in press)

18. Kamran, T: Coincidence and fixed points for hybrid strict contractions. J. Math. Anal. Appl. 299, 235-241 (2004)

19. Haghi, RH, Rezapour, S, Shahzad, N: Some fixed point generalizations are not real generalizations. Nonlinear Anal. 74, 1799-1803 (2011)

20. Banach, S: Sur les opérations dans les ensembles abstraits et leurs applications aux équations intégrales. Fundam. Math. 3, 133-181 (1922)

21. Al-Thagafi, MA: Common fixed points and best approximation. J. Approx. Theory 85, 318-323 (1996)

22. Al-Thagafi, MA, Shahzad, N: Noncommuting selfmaps and invariant approximations. Nonlinear Anal. 64, 2778-2786 (2006)

23. Al-Thagafi, MA, Shahzad, N: Coincidence points, generalized I-nonexpansive multimaps and applications. Nonlinear Anal. 67, 2180-2188 (2007)

24. Shahzad, N: A result on best approximation. Tamkang J. Math. 29, 223-226 (1998). Corrections: Tamkang J. Math. 30 165 (1999)

25. Shahzad, N: Invariant approximation and R-subweakly commuting maps. J. Math. Anal. Appl. 257, 39-45 (2001)

26. Shahzad, N: Invariant approximations, generalized /-contractions and R-subweakly commuting maps. Fixed Point Theory Appl. 1, 79-86 (2005)

27. Sintunavarat, W, Kumam, P: Coincidence and common fixed points for hybrid strict contractions without the weakly commuting condition. Appl. Math. Lett. 22, 1877-1881 (2009)

28. Latif, A, Bano, A: A result on invariant approximation. Tamkang J. Math. 33, 89-102 (2002)

29. O'Regan, $D$, Shahzad, N: Coincidence points and best proximity pair results for $R$-subweakly commuting multimaps. Demonstr. Math. 39, 845-854 (2006)

30. O'Regan, D, Shahzad, N: Coincidence points and invariant approximation results for multimaps. Acta Math. Sin. Engl. Ser. 23, 1601-1610 (2007)

10.1186/1687-1812-2014-112

Cite this article as: Sintunavarat et al:: Mizoguchi-Takahashi's type common fixed point theorems without $T$-weakly commuting condition and invariant approximations. Fixed Point Theory and Applications 2014, 2014:112 